\title{
Síndrome de Pallister-Killian en una paciente mestiza mexicana. Reporte de caso
}

\author{
Pallister-Killian syndrome in a Mexican mestizo patient. Case report
}

\author{
Dra. Paola Mendelsberg-Fishbein ${ }^{a}$, Dra. Constanza García-Delgado ${ }^{a}$ Biol. Linda B. Muñoz-Martínez $z^{a, b}$, \\ Dra. Maura Robledo-Cayetano ${ }^{a}$,Dr. Leonardo J. Mejía-Marín ${ }^{a}$,Dr. Luis E. Martínez-Barrera ${ }^{a}$, \\ Dra. Mabel Cerrillo-Hinojosa ${ }^{b}$ y Dra. Verónica F. Morán-Barroso
}

\section{RESUMEN}

El síndrome de Pallister-Killian es una entidad poco frecuente causada por tetrasomía 12p en mosaico. Presenta facies tosca, alopecia frontotemporal, frente prominente, fisuras palpebrales oblicuas ascendentes, hipertelorismo ocular, ptosis palpebral, estrabismo, epicanto, puente nasal ancho, nariz corta, narinas antevertidas, filtrum largo, labio superior delgado e inferior prominente, pabellones auriculares con lóbulos gruesos y protruidos, cuello corto, pezones supernumerarios, manos anchas, braquidactilia, alteraciones en la pigmentación de la piel, cardiopatía congénita, discapacidad intelectual y crisis convulsivas. Su diagnóstico es complejo, ya que, en sangre periférica, el cariotipo suele ser normal.

Se presenta el caso de una paciente mestiza mexicana de 4 años de edad con retraso en el desarrollo psicomotor y características fenotípicas que correspondieron a síndrome de Pallister-Killian. El cariotipo en fibroblastos de la biopsia de piel demostró mos47,XX,i(12)(p10)[85]/46,XX[21]. Un equipo multidisciplinario realiza el seguimiento con controles regulares por los departamentos de Neurología, Pediatría General y Genética Médica.

Palabras clave: síndrome de Pallister-Killian, tetrasomía 12p, mosaicismo.

\section{ABSTRACT \\ Pallister-Killian syndrome is caused by a tetrasomy $12 p$ mosaicism and is characterized by facial dysmorphism, pigmentary skin anomalies, congenital heart defects, diaphragmatic hernia, epilepsy and mental retardation. The diagnosis is complex as the cytogenetic analysis in blood is usually normal, requiring karyotyping in other tissues, therefore the clinical suspicion is critical to guide the diagnostic tests and the patient requires an interdisciplinary clinical evaluation regarding the several manifestation of the syndrome. We present the case of a Mexican mestizo female patient of 4 years of age referred by psychomotor delay and cleft palate; the clinical multidisciplinary evaluation demonstrated characteristics corresponding to the Pallister-Killian syndrome. The GTG}

a. Departamento de Genética, Hospital Infantil de México Federico Gómez.

b. Reproducción y genética AGN. Laboratorio de Genética, Hospital Ángeles del Pedregal. Ciudad de México, México.

Correspondencia:

Dra. Verónica F. Morán Barroso, vfmoran@himfg.edu.mx.

Financiamiento: Ninguno.

Conflicto de intereses: Ninguno que declarar.

Recibido: 1-6-2017

Aceptado: 7-9-2017 banding karyotype analysis was normal, the skin fibroblast was mos47, XX,i(12)(p10)[85]/46,XX[21]. This case is an example of the importance of the clinical evaluation in order to establish a diagnosis that is a challenge for the clinical multidisciplinary team to offer medical management and genetic counseling. Key words: Pallister-Killian syndrome, tetrasomy $12 p$, mosaicism.

http:/ / dx.doi.org/10.5546/aap.2018.e135

Cómo citar: Mendelsberg-Fishbein P, García-Delgado C, Muñoz-Martínez LB, et al. Síndrome de Pallister-Killian en una paciente mestiza mexicana. Reporte de caso. Arch Argent Pediatr 2018;116(1):e135-e138.

\section{INTRODUCCIÓN}

El síndrome de Pallister-Killian (SPK, OMIM 601803), también conocido como síndrome de Killian-Teschler-Nicola o síndrome de isocromosoma $12 \mathrm{p}$ en mosaico, fue descrito en 1977. ${ }^{1,2}$ Es causado por la presencia de un pequeño cromosoma metacéntrico supernumerario formado por dos brazos cortos o p del cromosoma 12 que ocasiona una tetrasomía de $12 \mathrm{p}$ en mosaico. ${ }^{1,3}$ El SPK tiene un espectro de manifestaciones clínicas muy amplio, que va desde la mortalidad perinatal a la supervivencia y a la adolescencia con discapacidad intelectual, dismorfias y cardiopatías. ${ }^{4,5}$ Se caracteriza por facies tosca, alopecia frontotemporal, cejas y pestañas escasas, frente prominente, fisuras palpebrales oblicuas ascendentes, hipertelorismo ocular, ptosis palpebral, estrabismo, epicanto, puente nasal ancho, nariz corta, narinas antevertidas, filtrum largo, labio superior delgado con el arco de cupido muy marcado, labio inferior prominente y retraso en la erupción dental. Los pabellones auriculares son grandes con lóbulos gruesos y protruidos, cuello corto, pezones supernumerarios, manos anchas, braquidactilia, polidactilia, acortamiento rizomélico de las extremidades ${ }^{6} \mathrm{y}$ alteraciones en la pigmentación de la piel con zonas de hipo- e hiperpigmentación. Puede asociarse a cardiopatía congénita (como persistencia del foramen oval, defectos septales atriales y ventriculares, y persistencia del conducto arterioso), ${ }^{7}$ hernia diafragmática, hipospadias, alteraciones renales, hipotonía, 
retraso en el desarrollo psicomotor y crisis convulsivas. ${ }^{1,2,4}$

La proporción del mosaicismo de la tetrasomía $12 p$ varía en los diferentes tejidos, ya que, en linfocitos de sangre periférica, puede estar ausente o en una proporción menor del $2 \%$; es más frecuente la línea celular anormal en fibroblastos de piel o de mucosa oral. ${ }^{7,8} \mathrm{La}$ variación del mosaicismo en los diferentes tejidos se ha atribuido a un efecto in vitro de los cultivos celulares en el que se pierden de manera preferencial las células con cromosomas alterados; sin embargo, el porcentaje de células anormales no se ha relacionado con la gravedad clínica. ${ }^{4,9}$ La presencia del isocromosoma extra se puede observar por cariotipo con bandas GTG (por la desnaturalización con tripsina y la tinción de Giemsa) o por medio de hibridación fluorescente in situ (fluorescent in situ hybridization; FISH, por sus siglas en inglés) con sondas específicas para la región $12 \mathrm{p}$. El análisis por microarreglos de tipo de hibridación genómica comparativa (array comparative genome hybridization; aCGH, por sus siglas en inglés) permite la identificación de la tetrasomía en sangre periférica y facilita, así, el diagnóstico en aquellos casos en los que la

\section{FIGURA 1. Fenotipo de la paciente a los dos años de edad}

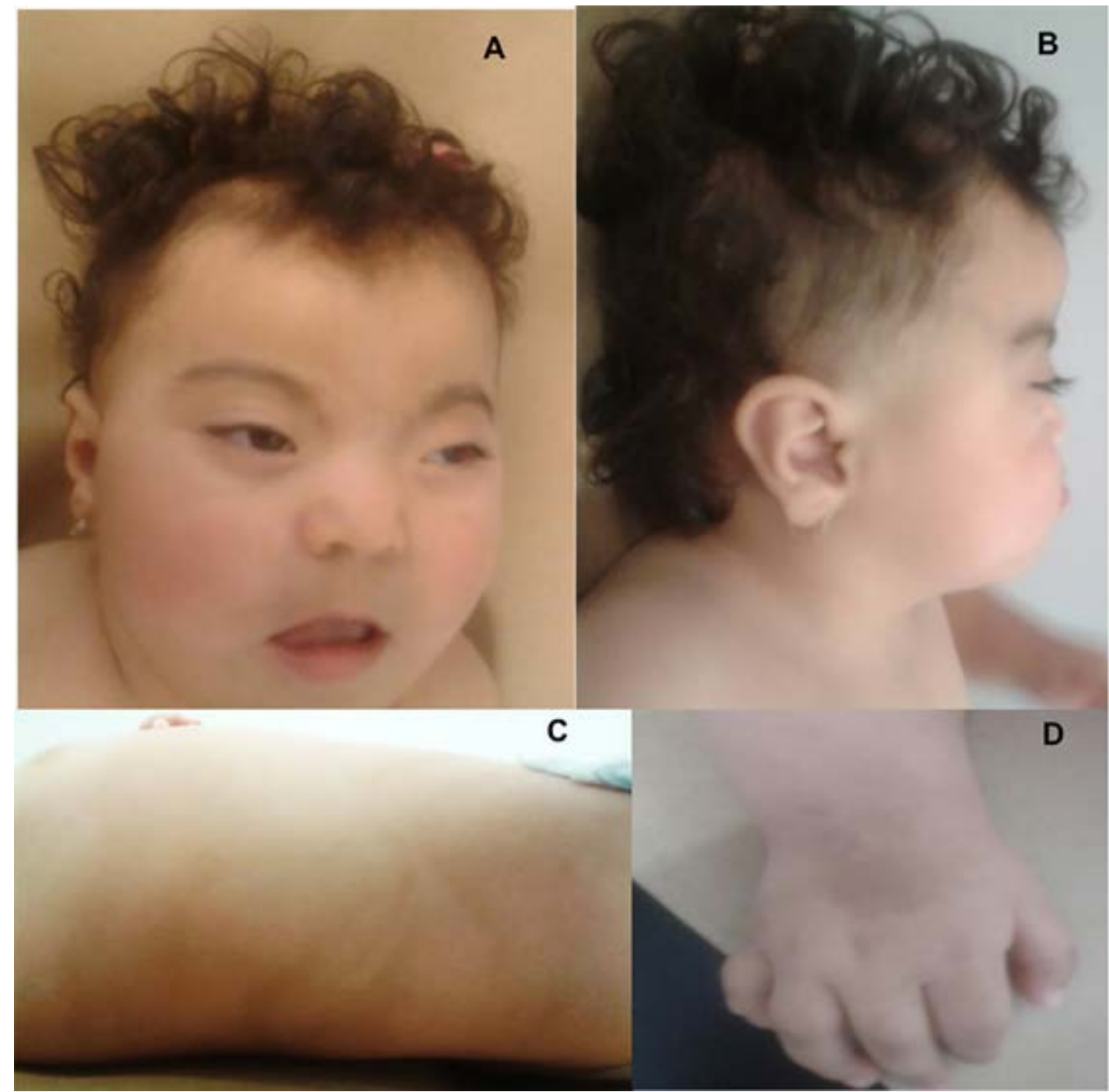

(A) Telecanto, hipertelorismo, puente nasal ancho, narinas antevertidas, fisuras palpebrales oblicuas ascendentes, filtrum largo, labio superior delgado, labio inferior prominente. (B) Pabellones auriculares de baja implantación, zonas de alopecia.

(C) Manchas hiperpigmentadas que siguen las líneas de Blaschko. (D) Braquicamptosindactilia en la mano 
sospecha clínica no lleva a la biopsia de piel para la obtención de fibroblastos. ${ }^{10,11}$

Se presenta el caso de una paciente con diagnóstico de SPK, quien fue referida por retraso del desarrollo y paladar hendido.

\section{CASO CLÍNICO}

Paciente femenina mestiza mexicana referida a los 2 años de edad, hija única de madre de 22 años de edad y padre de 25 años de edad al momento del nacimiento de la paciente, ambos sanos y no consanguíneos. La madre presentó una trombosis venosa en la pierna izquierda un mes antes del nacimiento. Nacida a las 37 semanas de gestación por vía vaginal; presentó una fractura de la clavícula izquierda por trauma obstétrico, que requirió hospitalización por dos días, ya que tuvo mala adaptación cardiopulmonar y dificultad respiratoria. Apgar 7 al minuto y 8 a los 5 minutos del nacimiento, peso de 2950 $\mathrm{g}$ (percentil 25), talla de $49 \mathrm{~cm}$ (percentil 50) y paladar hendido. Presentó retraso global del desarrollo, caracterizado por sostén cefálico a los 6 meses, sonrisa social a los 5 meses, rodó sobre su propio eje a los 12 meses, inició dentición a los 18 meses de vida. El estudio de tomografía axial computarizada a los 10 meses de edad demostró turricefalia, disminución del volumen del parénquima cerebral y aumento del espacio subaracnoideo; se diagnosticó hipoacusia profunda bilateral de tipo mixto; se le realizó palatoplastía a los 15 meses de edad. A los 17 meses de vida, se diagnosticó otomastoiditis bilateral y, a los 19 meses, se colocaron tubos de ventilación bilateral. El estudio ecocardiográfico a los dos años de edad reportó aorta trivalva con corazón estructuralmente sano. La paciente fue referida a la consulta de Genética por el antecedente de paladar hendido y retraso en el desarrollo psicomotor.

En la exploración física a los 2 años y 3 meses, presentó peso de $12 \mathrm{~kg}$ (percentil 50), talla de $85 \mathrm{~cm}$ (percentil 50), perímetro cefálico de $48 \mathrm{~cm}$ (percentil 10). Cráneo turricéfalo con alopecia parcial, dismorfias faciales con fisuras palpebrales oblicuas ascendentes, telecanto, hipertelorismo, puente nasal ancho, labio superior delgado, labio inferior prominente, paladar hendido completo, braquicamptosindactilia, manchas hipercrómicas que seguían las líneas de Blaschko en las extremidades superiores, las inferiores y la región lumbar (Figura 1). Se realizó cariotipo en sangre periférica con bandas GTG con un completo cromosómico 46,XX [25]. Por la sospecha clínica, se realizaron, adicionalmente, dos cariotipos en fibroblastos; el primero de piel sin hiperpigmentación, con resultado normal, y el segundo tomado de una de las manchas hipercrómicas ya descritas, cuyo resultado fue mos47,XX,i(12)(p10)[85]/46,XX[21] (Figura 2).

El seguimiento de la paciente incluyó el estudio de electroencefalograma a los 2 años y 7 meses, que demostró leve disfunción generalizada; resonancia magnética de cráneo a los 2 años y 8 meses, en la que se identificó un aumento en el espacio subaracnoideo de forma generalizada, adelgazamiento del cuerpo calloso a nivel de la silla turca con disminución del diámetro rostrocaudal, con abombamiento de la adenohipófisis hacia la cisterna supraciliar. A los 4 años, se realizó mastoidectomía derecha. La paciente ha estado en seguimiento con controles regulares por los departamentos de Neurología, Pediatría General y Genética Médica.
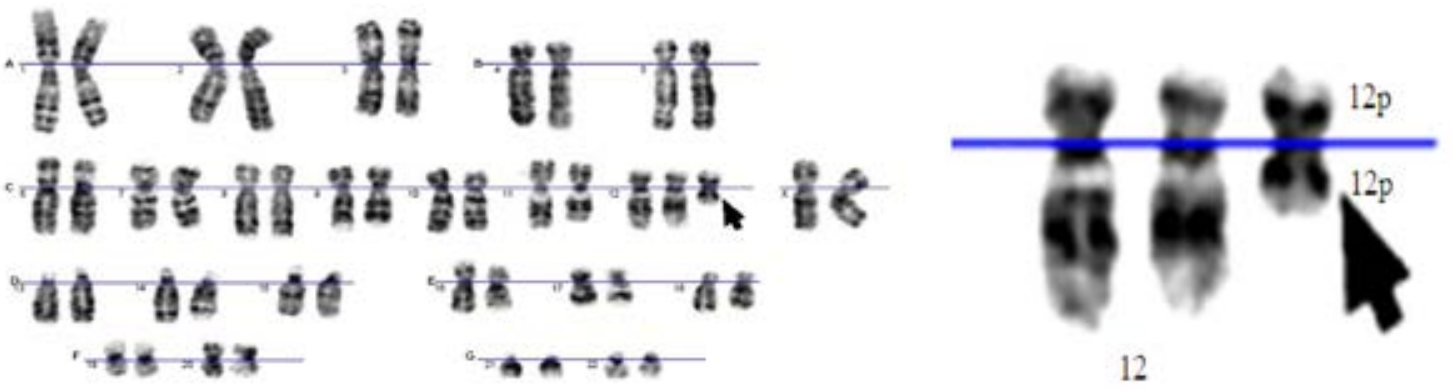

A) Se muestra el resultado 47,XX,i(12)(p10) y se indica el isocromosoma 12p (flecha).

B) Cariotipo parcial que muestra el par de cromosomas 12 y el isocromosoma 


\section{DISCUSIÓN}

El SPK es una entidad muy poco frecuente; se ha calculado una prevalencia de 1 en 20000 recién nacidos vivos. ${ }^{8}$ Su diagnóstico es complejo por ser una de las cromosomopatías en las que el reconocimiento de las características clínicas es esencial para su diagnóstico, ya que el cariotipo en sangre periférica suele ser normal y se deben realizar estudios citogenéticos en otros tejidos, especialmente, de piel que abarque una zona con hiperpigmentación. ${ }^{1,2}$

Nuestra paciente fue referida por presentar retraso en el desarrollo y paladar hendido; ambas situaciones se presentan con frecuencia en la práctica pediátrica. En el presente caso, la sospecha clínica se hizo por las características faciales y dermatológicas; sin embargo, la paciente no presentaba otras alteraciones descritas en el SPK, como la cardiopatía congénita o las alteraciones genitourinarias, polidactilia o acortamiento rizomélico de las extremidades. Tenía un estudio electroencefalográfico anormal, pero no ha presentado crisis convulsivas, si bien estas pueden tener una aparición más tardía. ${ }^{1,2}$

El SPK ha sido descrito con incidencia esporádica asociada con bajo riesgo de recurrencia. Se han propuesto varias hipótesis para explicar la formación del isocromosoma $12 p$ que sugieren un origen materno proveniente de una no disyunción en meiosis II. ${ }^{2}$ Se ha reportado un caso con microduplicación de la región 12 p13.31, que comprende 26 genes, 3 de los cuales se proponen como candidatos causantes del fenotipo: ING4, CHD4 y MAGP $2 .{ }^{1}$ Como diagnósticos diferenciales, se debe considerar la trisomía $12^{12}$ y el síndrome de Fryns ${ }^{8}$ (OMIM 229550).

Este caso ejemplifica la importancia de que el médico pediatra de primer contacto realice la evaluación clínica integral de los pacientes que le permita establecer una sospecha diagnóstica para considerar una etiología que incluya síndromes poco frecuentes, como el que nos ocupa, y, sobre la base de la clínica, descartar alteración cromosómica, la cual se demostró, en este caso, en el análisis de cariotipo en fibroblastos de piel realizado en las manchas hipercrómicas. Este caso representa un reto en la evaluación clínica interdisciplinaria con el objetivo de establecer la entidad patológica para otorgar manejo y asesoramiento genético.

\section{REFERENCIAS}

1. Schinzel A. Tetrasomy 12p (Pallister-Killian syndrome). J Med Genet 1991;28(2):122-5.

2. Pallister P. Pallister Killian syndrome: historical perspective and foreword. Am J Med Genet Part A 2012;158A(12):2999-3001.

3. Blyth M, Maloney V, Beal S, et al. Pallister-Killian syndrome: a study of 22 British patients. I Med Genet 2015;52(7):454-64.

4. Izumi K, Krantz ID. Pallister Killian syndrome. Am J Med Genet C Semin Med Genet 2014;166C(4):406-13.

5. Genevieve D, Cormier-Daire V, Sanlaville D, et al. Mild phenotype in a 15 year old boy with Pallister-Killian syndrome. Am J Med Genet A 2003;116A(1):90-3.

6. Toledo-Bravo de Laguna L, del Campo-Casanelles M, Santana-Rodriguez A, et al. Presentación de tres casos de síndrome de Pallister-Killian. Rev Neurol 2014;58(2):63-8.

7. Wilkens A, Liu H, ParkK, et al. Novel clinical manifestations in Pallister-Killian syndrome: comprehensive evaluation of 59 affected individuals and review of previously reported cases. Am J Med Genet A 2012;158A(12):3002-17.

8. Theisen A, Rosenfeld JA, Farrell SA, et al. aCGH detects partial tetrasomy of $12 \mathrm{p}$ in blood from Pallister-Killian syndrome cases without invasive skin biopsy. Am J Med Genet A 2009;149A(5):914-8.

9. Wenger SL, Boone LY, Steele MW. Mosaicism in Pallister i(12p) syndrome. Am J Med Genet 1990;35(4):523-5.

10. Ramírez-Fernández MA, García-Cavazos R, SánchezMartínez HF.Síndrome de Pallister-Killian. Comunicación de un caso. Ginecol Obstet Mex 2007;75(7):412-8.

11. Lee MN, Lee J, Yu HJ, et al. Using Array-Based Comparative Genomic Hybridization to Diagnose Pallister-Killian Syndrome. Ann Lab Med 2017;37(1):66-70.

12. Inage E, Suzuki M, Minowa K, et al. Phenotypic overlapping of trisomy 12p and Pallister-Killian syndrome. Eur J Med Genet 2010;53(3):159-61. 